The Wellcome Trust Sanger Institute (Hinxton, UK) has recently announced the discovery of mutations in the POT1 gene that appear to be responsible for a hereditary form of melanoma. Approximately one in every 20 patients diagnosed with melanoma has family history of the disease and identification of causative genetic factors such as this may allow identification of those who should be part of melanoma surveillance programs. The study appears online in Nature Genetics.

"Genomics is on the verge of transforming the healthcare system - this study highlights the potential clinical benefits that can be gained through genomic studies and offers potential strategies to improve patient care and disease management," commented David Adams, co-senior author from the Wellcome Trust Sanger Institute. "With this discovery we should be able to determine who in a family is at risk, and in turn, who should be regularly screened for early detection."

Previously identified mutations such as deleterious germline variants in $C D K N 2 A$ and variants in $C D K 4, B R C A 2$ and $B A P 1$ account for approximately $40 \%$ of inherited melanoma. In an attempt to identify new high-penetrance susceptibility genes that might be involved in the other $60 \%$ of cases, the team sequenced the genomes of 184 individuals with hereditary melanoma of unknown genetic cause.

Their results indicated that those with inactivating mutations in POT1 were at an extremely high risk of developing melanoma. It was further established that the mutations prevent POT1 from maintaining telomere length and integrity. Evidence of the mutations was also noted in some cases of other cancer types in families, including leukemias and brain tumors.

"Our research is making a real difference to understanding what causes melanoma and ultimately therefore how to prevent and treat melanoma, and is a prime example of how genomics can transform public health," explained Julia Newton Bishop, co-senior author from the University of Leeds (UK). "This study would not have been possible

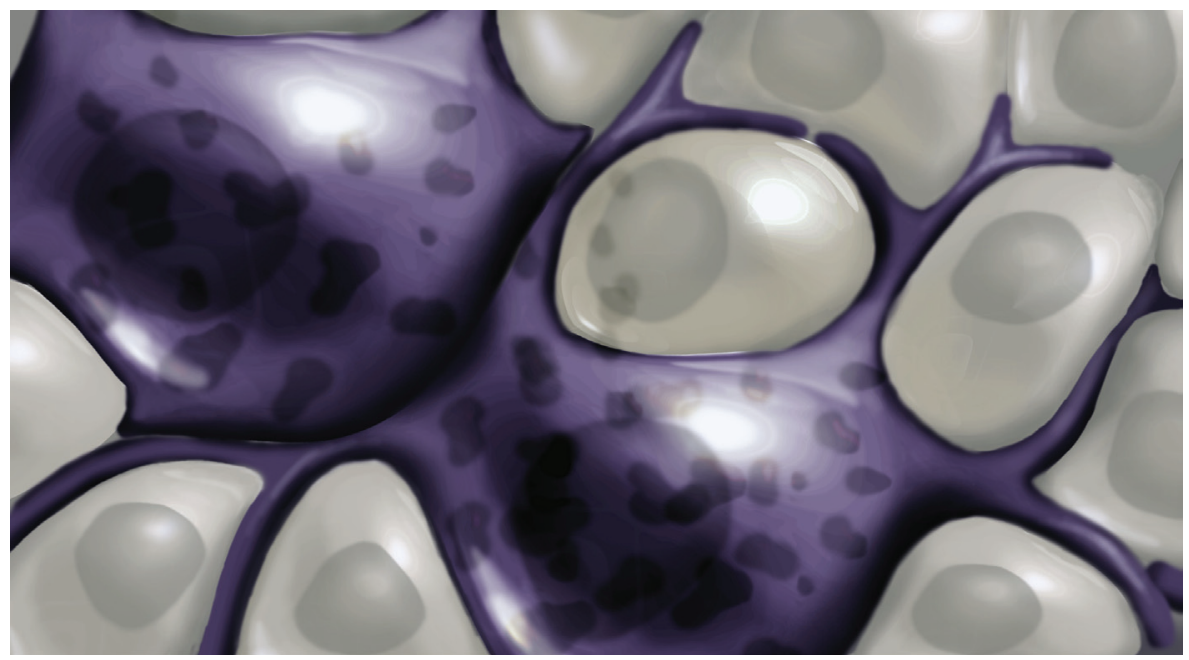

\section{in the news...}

- Lead story: New high-risk gene mutation for hereditary melanoma development identified

- Predicting prognosis in cancers of the lung and head and neck with a novel biomarker pg 1118

- Leukemia microarrays for a more definitive prognosis and treatment? pg 1118

- New targeted cancer therapy for resistant gynecologic cancers pg 1119

- Priority Paper Alerts pg 1120
Future : Medicine part of 
without the help and patience from the families that suffer from these devastating, inherited forms of melanoma."

The researchers plan to further their work into POT1 variants and melanoma predisposition by developing cells and mice with an inactive POT1 gene in which to test potential drug therapies.
- Written by Emily Brown

Sources: Robles-Espinoza CD, Harland M, Ramsay AJ et al. POT1 loss-of-function variants predispose to familial melanoma. Nat. Gen. doi:10.1038/ng.2947 (2014) (Epub ahead of print); Wellcome Trust Sanger Institute press release: www.nature.com/ng/journal/ vaop/ncurrent/full/ng.2947.html

Also available at www.oncology-central.co.uk

\section{Predicting prognosis in cancers of the lung and head and neck with a novel biomarker}

A new biomarker thought to be associated with better outcomes in both head and neck cancer and non-small-cell lung cancer (NSCLC) has recently been identified by a team led by individuals from The Scripps Research Institute (FL, USA). The finding, published this week online ahead of print in Cancer, may assist in the development of new therapies and help physicians determine the best treatments for these patients.

The work included immunohistochemistry examination of samples from 187 patients with NSCLC and 60 patients with head and neck squamous cell carcinomas in order to assess CCT $\alpha$ expression and association with patient outcomes. The study concluded that high expression of CCT $\alpha$, an antigen that prompts an immune response, is prognostic of survival in these individuals.

Expression of the DNA repair protein ERCC1 is being investigated in many clinical trials as a determinant of whether patients with many cancer types (including lung, pancreatic, gastric, colorectal, esophageal and ovarian) should be treated with platinum-based therapy and is suggested to be prognostic of survival in NSCLC patients. The current works suggests that positive results for ERCC1 expression may actually be due to expression of CCT $\alpha$, to which the anti-ERCC1 monoclonal antibody $8 \mathrm{~F} 1$ also binds.

"Based on what we found, a high CCT $\alpha$ expression appears to be indicative of survival, making CCT $\alpha$ a promising biomarker," commented Laura Niedernhofer (The Scripps Research Institute), one of the study leads. "Our findings suggest that CCT $\alpha$ may, in fact, be more important in determining outcomes in patients with both types of cancer than the already established ERCC1.”

\section{- Written by Emily Brown}

Sources: Vaezi AE, Bepler G, Bhagwat NR et al. Choline phosphate cytidylyltransferase-a is a novel antigen detected by the anti-ERCC1 antibody 8F1 with biomarker value in patients with lung and head and neck squamous cell carcinomas. Cancer doi:10.1002/cncr.28643 (2014) (Epub ahead of print); The Scripps Research Institute press release: www.scripps.edu/news/ press/2014/20140402niedernhofer.html

Also available at www.oncology-central.co.uk

\section{Leukemia microarrays for a more definitive prognosis and treatment?}

Findings presented last week at the American College of Medical Genetics and Genomics Annual Clinical Genetics Meeting (held on 25-29 March 2014 in Nashville, TN, USA) indicate that some patients with cytogenetically normal acute myelogenous leukemia (AML) have a distinct pattern of genetic abnormalities that could better define their prognosis and treatment.
AML represents the most common acute leukemia of adulthood and occurs when a disproportionate number of stem cells remain in the blast phase, rather than maturing to white blood cells. Patients with disease that appears cytogenetically normal have high percentages of cancer-producing cells called blasts in their blood and bone marrow, but do not show the distinctive chromosomal alterations that typically help 
characterize the leukemia. Furthermore, these individuals demonstrate widely varying outcomes after treatment for AML, typically including chemotherapy and bone marrow transplants.

"(Cytogenetically normal patients) show a normal chromosomal picture but they are clearly sick," commented Ravindra Kolhe of the Medical College of Georgia at Georgia Regents University (GA, USA). In order to investigate potentially causative genes in this subtype, Kolhe partnered with Affymetrix (CA, USA) to look directly at the genes within chromosomes using CytoScanHD microarray technology.

After analyzing patient samples with 2.7 million genetic probes, a unique and distinct pattern of gene expression was found in 22 patients with cytogenetically normal AML. The microarrays identified small, previously undetectable changes in the DNA of these patients who had disease with apparently normal chromosomes.

With microarray technology slowly developing a clinical presence, Kolhe is continuing with collection of patient data and is investigating these unique genetic changes in mice to confirm whether they cause leukemia. Further identifying the causative genes could lead to a more targeted therapy and definitive prognosis for these individuals, Kolhe said.

- Written by Emily Brown

Source: Georgia Regents University press release: http://news.gru.edu/archives/11788

Also available at www.oncology-central.co.uk

\section{New targeted cancer therapy for resistant gynecologic cancers}

Mutations in the infamous $B R C A$ gene are synonymous with breast cancer and in addition women who carry a $B R C A$ mutation also have a high risk of developing gynecologic cancers. A recent Phase II study, presented at the Society of Gynecologic Oncology (SGO) Annual Meeting on Women's Cancer (FL, USA), has shown that gynecologic cancer cells that have a $B R C A$ mutation appear to be sensitive to veliparib.

This drug works by targeting an enzyme called PARP, which is essential in enabling cancer cells to repair themselves after DNA damage. As such veliparib has been effective when combined with chemotherapy, but it had not be identified if the drug is effective when used as a single agent, until this recent trial.

Robert Coleman, lead author of the study and professor and vice chair of clinical research at the University of Texas MD Anderson Cancer Center (TX, USA), explained that "One criticism of the PARP drugs is they are not active in patients who have developed resistance to other therapies, but we found veliparib appears to be effective in some platinum-resistant patients with recurrent or persistent disease." Coleman continued: "Most of these patients have run out of treatment options, and it is very hopeful to potentially have another therapy to offer them."

A twice-daily dose of veliparib was given to 50 patients with $B R C A$ gene mutations. Disease was stabilized for more than 4 months in over half of the patients (26) and overall $26 \%$ of patients responded positively to the treatment, with their tumors reducing in size. In addition, tumors in two patients completely disappeared.

Coleman concluded: "Patient recruitment can be a problem for many clinical trials, however, this one filled up very quickly, which reflects that women and their doctors understand that PARP inhibitors hold real promise."

- Written by Theo Bond

Source: The Society of Gynecologic Oncology press release: www.sgo.org/newsroom/news-releases/targetedcancer-therapy-may-treat-resistant-gynecologic-cancers 


\section{BULLETIN BOARD}

\section{Priority Paper Alerts}

Delgado O, Batten KG, Richardson JA et al. Radiation-enhanced lung cancer progression in a transgenic mouse model of lung cancer is predictive of outcomes in human lung and breast cancer. Clin. Cancer. Res. 20(6), 1610-1622 (2014). This study aimed to determine the impact of radiation exposure on lung cancer progression in vivo, and extrapolate this to human carcinogenesis. K-ras(LA1) mice were irradiated with varying doses, with microarray analyses were performed on whole lung tissue 70-days later. These were compared with age-matched unirradiated controls. Survival analyses were performed and comparative genomic analysis performed. Radiation exposure accelerated progression by affecting inflammatory pathways, and results predicted that a similar mechanism will occur with human lung and breast carcinogenesis.

Morfouace M, Shelat A, Jacus $M$ et al. Pemetrexed and gemcitabine as combination therapy for the treatment of group 3 medulloblastoma. Cancer Cell doi:10.1016/j.ccr.2014.02.009 (2014) (Epub ahead of print). Using a high-throughput cell-based assay to screen 7389 compounds in mouse G3 MB neurospheres, researchers found that pemetrexed and gemcitabine inhibited G3 MB proliferation in vitro and in vivo. In combination, they increased survival in mice bearing human group 3 MYC-overexpressing medulloblastoma compared with those left untreated. The researchers reported that the concentrations used are safe and achievable in patients.

\section{About the Bulletin Board}

The News highlights some of the most important events and research. If you have newsworthy information, please contact:

Francesca Lake, Commissioning Editor, Future Oncology

Future Medicine Ltd, Unitec House, 2 Albert Place, London, N3 1QB, UK

f.lake@futuremedicine.com 